\title{
Lohnende Anpassungen
}

\begin{abstract}
Die Integration des betrieblichen Umweltcontrollings in die bestehenden betriebswirtschaftlichen Informationssysteme gehört zu den wichtigen Herausforderungen eines integrierten Umweltmanagements. Was sind die Möglichkeiten und Grenzen der Integration des Konzepts der Flusskostenrechnung in das weit verbreitete System SAP R/3? Wie können damit Reststoffströme mengen- und wertmäßig erfasst und ausgewertet werden? Die vorhandenen Standardfunktionalitäten des SAP R/3 lassen sich hierfür gut ausnutzen.
\end{abstract}

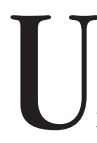

Von Carl Ulrich Gminder und Stefan Frehe mweltmanagement und Umweltcontrolling haben sich in vielen Unternehmen in den 90er Jahren etabliert. Trotzdem nimmt das Umweltkostenmanagement in den meisten Unternehmen keine oder nur eine untergeordnete Stellung ein. Dabei ist gerade dieses in der Lage, das Argument vieler Unternehmen, „Umweltschutz verursacht nur Kosten", zu widerlegen. In Praxisanwendungen konnten Kosteneinsparpotenziale mittels Einführung eines Umweltkostenmanagements aufgedeckt und auch genutzt werden.

Besonders bedeutsam für erfolgreiches Umweltkostenmanagement ist die Integration in die betrieblichen Informationssysteme, die von den klassischen Abteilungen wie Einkauf, Produktion, Vertrieb und Finanzen/Controlling genutzt werden. Diese Systeme liefern das Fundament betrieblicher Daten in Form von Mengen- und Wertegerüsten, jedoch auf betriebswirtschaftliche Fragestellungen ausgerichtet. Daten für das Umweltcontrolling sind zwar vorhanden, aber selten in einer nutzbaren Struktur. Die Beschaffung, Pflege und Auswertung von Daten für das Umweltcontrolling ist meist zusätzlich zu leisten. Ziel für ein erfolgreiches Umweltkostenmanagement ist daher zum einen, diesen Aufwand zu vermeiden, und zum anderen, die Auswirkungen von Umweltbe- und -entlastungen auf bestehende Werteflüsse transparent zu machen.

\section{Die Flusskostenrechnung}

Als Basis der Betrachtung wird hier der Umweltkostenansatz der Flusskostenrechnung (FKR) gewählt. Als Vollkostenrechnung konzipiert, konzentriert sich die FKR auf die mengen- und wertespezifische Verfolgung von Stoff- und
Energieflüssen (S+E-Flüsse) im Unternehmen von der Beschaffung der Rohstoffe über die Fertigung der Produkte bis hin zur Entsorgung der Reststoffe. Die S+E -Flüsse werden als Kostenträger gesehen, die für die entstehenden Umweltwirkungen verantwortlich sind. Die durch die FKR gewonnenen Informationen dienen dem operativen Management, um Maßnahmen zur Senkung von Kosten und Umweltbelastungen im Unternehmen realisieren zu können. Zur Auswertung bietet der Ansatz der FKR unter anderem verschiedene Verfahren zur verursachungsgerechten Verrechnung der Reststoffmaterialkosten auf Kostenstellen an (1).

\section{Integration in SAP R/3}

SAP R/3 ist eines der gebräuchlichsten betrieblichen Informationssysteme. Ziel der Integration einer Flusskostenrechnung in SAP R/3 ist die Ausnutzung und Anpassung der dort vorhandenen Standardfunktionalitäten. Damit kann sowohl auf Zusatzentwicklungen und Programmmodifikationen in $\mathrm{R} / 3$, wie etwa das bestehende Umweltmanagement-Modul, als auch auf externe Programme, die über Schnittstellen mit dem R/3 verbunden sind, verzichtet werden.
Da die Flusskostenrechnung einen sehr umfassenden Kostenmanagement-Ansatz darstellt, wurden für die Prüfung der Integration in SAP $\mathrm{R} / 3$ Schwerpunkte auf folgende Arten von Reststoffen gesetzt:

- Ausschuss, das heißt Endprodukte, die nicht den Qualitätsanforderungen entsprechen und nicht nachgearbeitet oder entsorgt werden,

- Nebenprodukte, die beim Produktionsprozess als Abfälle anfallen und wiederaufbereitet oder entsorgt werden müssen.

- Als Differenzierungskriterien dienen dabei die Eigenschaften planbar und nicht planbar. In Abbildung 1 ist die Differenzierung der Reststoffarten in einer Matrix zusammengefasst.

\section{- Planbare Ausschüsse}

Dieser Ansatz geht davon aus, dass die während der Produktion anfallenden Ausschüsse in ihrem mengenmäßigem Umfang aufgrund bestehender Erfahrungen aus dem Produktionsbetrieb und verfahrenstechnischen Restriktionen bekannt sind. Sie können damit vor Produktionsbeginn im SAP-System berücksichtigt werden. Im SAP Standard werden die Herstellkosten eines planbaren Ausschusses berïcksichtigt, indem die Produktkalkulation ein modifiziertes Mengengerüst aus den Ausschussinformationen erstellt. Diese Ausschussdaten werden als Komponenten-, Baugruppen- oder Vorgangsausschuss in den jeweiligen Stammdaten des Arbeitsplanes bzw. des Materials erfasst. Der Mengen- und Leistungsverbrauch wird gemäß des neuen Mengengerüstes kalkuliert. Die entstandenen Ausschusskosten werden dabei den entsprechenden Kostenelementen zugeordnet, so dass eine kostenartengerechte Aufschlïsselung und damit ein preview der zu erwartenden Kosten ermöglicht wird - eine separate Zuordnung zum Material planbarer Ausschuss erfolgt jedoch nicht.

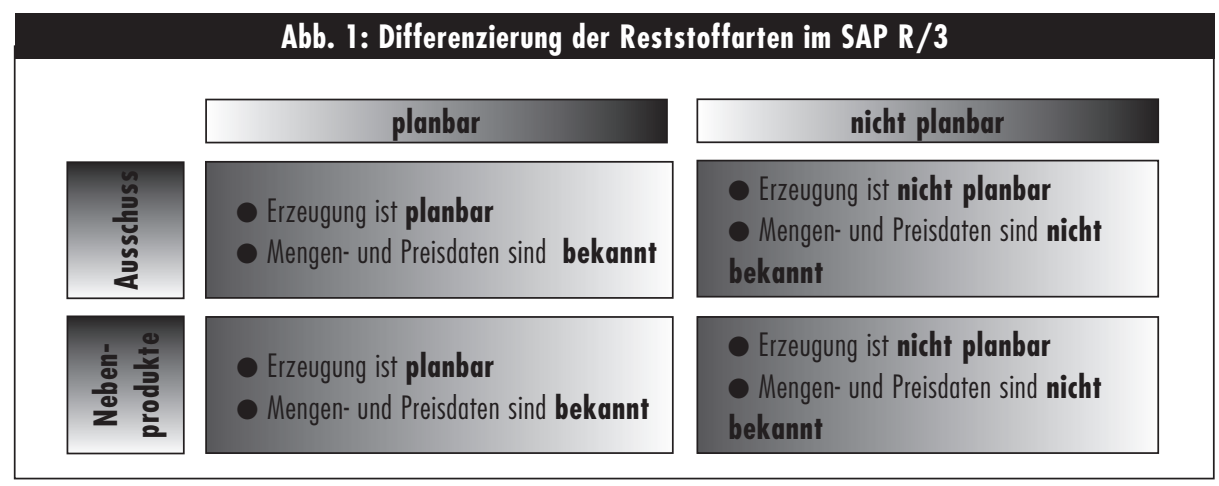


Die Vorgehensweise in SAP aus Sicht der Flusskostenrechnung ist also mit Einschränkungen verbunden: Zum einen geht das Material ,Ausschuss" als Kostenträger verloren, da es als solcher im Controlling nicht weiter verfolgt wird, und zum anderen ist eine verursachungsgerechte Zuordnung der entstandenen Kosten auf Kostenstellen kaum mehr möglich. Da es im Standard ohne Zusatzentwicklungen nicht möglich ist, planbare Ausschüsse flusskostengerecht zu erfassen und auszuwerten, sind Anpassungen zur Behandlung von geplantem Ausschuss im SAP R/3 vorzunehmen. Dazu greift man auf die Vorgehensweise zur Abbildung nicht planbarer Ausschüsse zurück (siehe Abschnitt weiter unten). Dieses Verfahren ist in der Lage einen detaillierten Überblick über die Flusskosten auch für die planbaren Ausschüsse sicherzustellen.

\section{- Planbare Nebenprodukte}

Planbare Nebenprodukte können in SAP R/3 flusskostengerecht erfasst und ausgewertet werden. Im Vorfeld sollte die Detailgenauigkeit der Flusskosten-Informationen festgelegt werden, da sich daraus der Aufwand für die Systemarbeiten bestimmt.

Die vorgehensweise für diese Reststoffart setzt voraus, dass als Vorleistung die stoffliche Zusammensetzung dieser Nebenprodukte und das daraus resultierende Mengengerüst ermittelt werden. So kann sich zum Beispiel Abwasser aus Wasser, Tensiden und Schwermetallen zusammensetzen, die bereits als Input für die Herstellung des Produktes benötigt werden. Die Materialeinzelkosten der einzelnen Bestandteile sind aufgrund der Datenintegration zum Modul Materialwirtschaft verfügbar und können in die Flusskostenbetrachtung einfließen.

Wird nun ein SAP-Fertigungsauftrag (FAUF) angelegt, so werden die Mengen- und die Kostendaten sowohl des Produktes als auch des Nebenproduktes automatisch vom System ermittelt. Als Ergebnis erhält man eine Sicht auf die Materialkosten des Nebenproduktes, die herstellkostenmindernd wirken.

Ist man an der genauen Aufsplittung der einzelnen Kostenarten interessiert, stehen wiederum Standardkalkulationen zur Verfügung. Für weitere Kostenzuordnungen, wie interne Entsorgungskosten, kann analog zum planbaren Ausschuss verfahren werden.

\section{- Nicht planbarer Ausschuss}

Nicht planbarer Ausschuss wird im SAP-Standard innerhalb der Auftragsrïckmeldungen nur zu Berichtszwecken als statistische Kennzahl erfasst und nicht weiter aus der Sicht der Kostenrechnung verfolgt. Als Lösung wird deswegen vorgeschlagen, mittels eines separat angelegten Fertigungsauftrages die Kosten zu erfassen, die mit der „Herstellung“ des Ausschusses angefallen sind. Dieser Auftrag muss nicht immer neu angelegt werden, sondern wird lediglich wieder aufgerufen, wenn während der Produktion nicht planbarer Ausschuss anfällt. Der Ausschuss wird auf diesen FAUF zurückgemeldet.

Als Ergebnis erhält man durch die Daten auf dem Ausschussauftrag einen Überblick über die Kosten, die die Produktion des Ausschusses verursacht hat. Je nach Ausgestaltung des Arbeitsplanes können auch Kosten, wie beispielsweise interne Transportkosten, auf dem FAUF gesammelt werden. Zusätzlich angefallene interne Entsorgungskosten können unter Verwendung der innerbetrieblichen Leistungsverrechnung dem Ausschussauftrag direkt belastet werden. Somit wird auch eine kostenträgergerechte Zuordnung dieser Umweltkosten erreicht.

Über eine Anpassung bestehender Abrechnungsschemata können die gesammelten Kosten nach Flusskostenarten differenziert an die verursachenden Kostenstellen abgerechnet werden und stehen den Kostenstellenanalysen zur Verfügung.

\section{Nicht planbare Nebenprodukte}

Bei den nicht planbaren Nebenprodukten handelt es sich hier um Reststoffe, die in keinem Zusammenhang zu einem Kostenträger stehen und losgelöst davon zu behandeln sind. Aus diesem Grund gestaltet sich die Zuordnung der einzelnen Flusskosten zu diesen Reststoffen als problematisch.

Dies liegt vor allem in der fehlenden Integration dieser Reststoffarten und deren Handling im SAP $\mathrm{R} / 3$ begründet. Im Kontext einer Flusskostenrechnung muss deshalb systemintern eine vertretbare Lösung gefunden werden. Als Instrument des Controllings bietet sich die manuelle Erfassung der Kosten auf einen speziell dafür angelegten Kostensammler an, zum Beispiel in Gestalt eines Innenauftrages. Fällt etwa ad hoc Abwasser an, so müssen bei der manuellen Erfassung der angefallenen Kosten Buchungen von der Kostenstelle Betriebsstoffe und eventuell einer beteiligten Bearbeitungskostenstelle auf einen Innenauftrag erfolgen. Die Materialkosten des Nebenproduktes lassen sich problemlos ermitteln, wenn die entsprechenden Stammdaten im System gepflegt sind. Eine verursachungsgerechte Zuordnung der Kosten zu den Kostenstellen ist über die zuvor erwähnten neuen Abrechnungsschemata möglich. Diese Lösung ist hinsichtlich der Transparenz der Flusskosten nicht vollständig, da beispielsweise Fertigungseinzelkosten in diesem Fall nur schwer lokalisier- und erfassbar sind.

\section{Schlussfolgerungen}

Die Detailanalyse der Funktionen des SAP-Standards zeigt, dass sich planbare Ausschüsse und Nebenprodukte sowie nicht planbare Ausschüsse in SAP R/3 abbilden und integrieren lassen. Um nicht planbare Nebenprodukte kosten- und wertmäßig zu erfassen, ist erhöhter manueller Aufwand erforderlich. Die Anpassungen des SAP-Systems für die Flusskostenrechnung konzentrieren sich einerseits auf die Pflege von Stammdaten der verschiedenen Reststoffarten sowie der zugehörigen Stücklisten und Arbeitspläne und andererseits auf die Anpassung der Kostenstellenstruktur sowie der zugehörigen Stammdaten.

Der Pflegeaufwand im SAP-System hängt in diesem Zusammenhang signifikant von den Anforderungen des Unternehmens hinsichtlich Detaillierungsgrad der geforderten Umweltinfor- mationen ab. Wichtig ist auch die Bereitschaft, bestehende Strukturen innerhalb der Systeme zu überdenken und sich einer ökologisch-flusskostenorientierten Sichtweise auf bestehende S+E-Flüsse zu öffnen.

\section{Anmerkung}

(1) Zu Details des Konzepts vgl. zum Beispiel Loew, Thomas/ Jürgens, Gunnar: Flusskostenrechnung versus Umweltkennzahlen, in: Ökologisches Wirtschaften 5-6/99, S. 27-29, und die dort angegebene Literatur.

\section{Die Autoren}

Carl Ulrich Gminder und Stefan Frehe arbeiten als Unternehmensberater im Bereich Chemie und Phar$\mathrm{ma} /$ Umweltmanagement bei der IDS Scheer AG. Kontakt: D-66115 Saarbruecken, Tel. 0681/2101300, Fax -1301, E-mail: u.gminder@ids-scheer.de, s.frehe@ids-scheer.de 
(c) 20I0 Authors; licensee IÖW and oekom verlag. This is an article distributed under the terms of the Creative Commons Attribution Non-Commercial No Derivates License (http://creativecommons.org/licenses/by-nc-nd/3.o/), which permits unrestricted use, distribution, and reproduction in any medium, provided the original work is properly cited. 